\title{
Mortality and life expectancy trends for male pensioners by pension income level
}

\author{
Juan Manuel Pérez-Salamero González \\ Department of Financial Economics and Actuarial Science, University of Valencia \\ Marta Regúlez Castillo \\ Department of Quantitative Methods. University of the Basque Country UPV/EHU) \\ Carlos Vidal-Meliá \\ Department of Financial Economics and Actuarial Science, University of Valencia. \\ Instituto Complutense de Análisis Económico, (ICAE), University of Madrid, Spain \\ and Centre of Excellence in Population Ageing Research (CEPAR), UNSW, \\ Sydney, Australia
}

Instituto Complutense de Análisis Económico
Keywords: Inequalities, Life Expectancy, Life Tables, Mortality, Socioeconomic Factors.

JEL Classification: C81, H55, 114, J26

A significant inverse relationship between mortality risk and different socioeconomic groups has almost always found. This paper deals with the case of Spain, since very little evidence concerning retirement pensioners is available for this country. We draw on the Continuous Sample of Working Lives (CSWL) to investigate the differences in socioeconomic mortality among retired men aged 65 and above over the longest possible period covered by this data source: 2005-2018. The only indicator of socioeconomic status we use is the amount of the initial pension of the retired population. For 2005-2010 we find a gap in life expectancy of 1.49 years between pensioners in the highest and lowest income groups. This gap widens over time and reaches 2.58 years for the period 20152018. The increase in life expectancy inequality cannot be attributed to the pension system reforms carried out over the period 2011-2013, given that the system has become more redistributive and there has been a clear increase in real terms in the amounts of minimum pensions over recent years. The causes might be traced back to the decrease in public spending on health over the period 2009-2018 and the increased spending on private health, which would presumably be of more benefit to those retirees with bigger pensions.

\section{ICAE Working Paper no 2102}

\section{February, 2021}

ISSN: 2341-2356

WEB DE LA COLECCIÓN:

http://www.ucm.es/fundamentos-analisis-economico2/documentos-de-trabajo-del-icae

Copyright $(2021$ by ICAE.

Working papers are in draft form and are distributed for discussion. It may not be reproduced without permission of the author/s. 


\title{
Mortality and life expectancy trends for male pensioners by pension income level
}

\author{
Juan Manuel Pérez-Salamero González
}

Department of Financial Economics and Actuarial Science, University of Valencia, Avenida de los Naranjos s.n., 46022 Valencia. (Spain). (e-mail: juan.perez-salamero@uv.es)

ORCID Author ID: 0000-0001-7710-4869

\section{Marta Regúlez Castillo}

Department of Quantitative Methods. University of the Basque Country (UPV/EHU). Avda. Lehendakari Aguirre 84, 48015 Bilbao (Spain). (e-mail: marta.regulez@,ehu.eus).

ORCID Author ID: 0000-0002-4694-5144

\section{Carlos Vidal-Meliá (Corresponding autor)}

Department of Financial Economics and Actuarial Science, University of Valencia, Avenida de los Naranjos s.n., 46022 Valencia. (Spain). Instituto Complutense de Análisis

Económico, Complutense (ICAE), University of Madrid, Spain (research affiliate) and Centre of Excellence in Population Ageing Research (CEPAR), UNSW, Sydney, Australia (research affiliate). (e-mail: carlos.vidal@uv.es). ORCID Author ID: 0000-0002-7227-5076.

\begin{abstract}
We draw on the Continuous Sample of Working Lives (CSWL) to investigate the differences in socioeconomic mortality among retired men aged 65 and above over the longest possible period covered by this data source: 2005-2018. This paper deals with the case of Spain, since very little evidence concerning retirement pensioners is available for this country. The only indicator of socioeconomic status we use is the amount of the initial pension of the retired population. For 2005-2010 we find a gap in life expectancy of 1.49 years between pensioners in the highest and lowest income groups. This gap widens over time and reaches 2.58 years for the period 2015-2018. The increase in life expectancy inequality cannot be attributed to the pension system reforms carried out over the period 2011-2013, given that the system has become more redistributive and there has been a clear increase in real terms in the amounts of minimum pensions over recent years. The causes might be traced back to the decrease in public spending on health over the period 20092018 and the increased spending on private health, which would presumably be of more benefit to those retirees with bigger pensions.
\end{abstract}

Key words: Inequalities, Life Expectancy, Life Tables, Mortality, Socioeconomic Factors.

JEL: C81, H55, I14, J26.

Transparency declaration. The corresponding author, in the name of the rest of the signatories, declares that the data and information contained in the study are precise, transparent and honest; that no relevant information has been omitted; and that all the discrepancies among authors have been adequately resolved and described.

Authors' contributions: Data curation, J.M.P.-S.G.; formal analysis, M.R.-C. and C.V.M.; investigation, J.M.P.-S.G., M.R.-C. and C.V.-M.; methodology, C.V.-M.; software, J.M.P.-S.G. and M.R.-C.; writing-original draft, M.R.-C. and C.V.-M.; writing-review and editing, J.M.P.-S.G., M.R.-C. and C.V.-M. 
Funding: The authors are grateful the financial assistance received from Spanish Ministry of the Economy and Competitiveness (Ministerio de Economía y Competitividad) projects ECO2015-65826-P, RTI2018-097087-B-100, Generalidad Valenciana (Valencian Government) project AICO/2019/075 and Basque Government project IT1336-19.

Acknowledgements: The authors are especially grateful to Manuel Ventura-Marco, who made an active contribution to previous versions, and to Peter Hall for his help with the English text.

Conflicts of interest/competing interests: The authors declare no conflict of interest.

Availability of data and material: Ethics approval is not required to use CSWL; its use for scientific purposes is regulated since inception. Researchers can request versions of the CSWL by post. A separate request must be made for each version. Requests consist of a user profile describing the project being carried out and a document accepting the CSWL's conditions of use. These are available at the following address:

http://www.seg-

social.es/wps/portal/wss/internet/EstadisticasPresupuestosEstudios/Estadisticas/EST21 1 


\section{Mortality and life expectancy trends for male pensioners by pension income level}

\section{1.-Introduction}

The connection between income and mortality could have implications for a wide range of social security regulations (Duggan et al., 2008). The literature almost always reports a significant inverse relationship between mortality risk and socioeconomic groups. Discussion of these inequalities has tended to focus more on younger rather than older age groups. However, there is a growing literature on the subject and more researchers seem to be taking an interest in inequalities in mortality related to socioeconomic status in elderly populations (Huisman et al., 2004).

Although the welfare state and welfare spending may have grown, health inequalities persist. And it is not those countries with more generous welfare states - such as those in Scandinavia - that present fewer health inequalities in relative terms, but countries like Italy, Spain and Portugal (Mackenbach, 2017; Mackenbach et al., 2008). This paradox is known as the public health puzzle (Bambra, 2011).

A substantial decline in mortality in lower socioeconomic groups has been reported in most of the European countries analysed in the literature (as cited below), (Mackenbach et al., 2016). However, relative inequalities in mortality have increased almost everywhere because percentage reductions are usually smaller in lower than in higher socioeconomic groups.

The literature reviewed which covers the UK (Longevity Science Panel, 2018, 2020), the USA (Waldron, 2007; Bosley et al., 2018), Italy (Belloni et al., 2013; Lallo \& Raitano, 2018), Canada (Adam, 2012; Wen et al., 2020), Germany (Kibele, 2013; Tetzlaff et al., 2020; Wenau et al., 2019), and the Netherlands (Kalwij et al., 2013), generally indicates that mortality gaps by socioeconomic status have not remained constant over time and that inequalities in mortality measured by socioeconomic status have increased in recent years.

This paper looks at the case of Spain, since there is still very little evidence concerning retirement pensioners in this country. To the best of our knowledge, only one paper so far has focused on the inequalities in mortality in connection with socioeconomic status in the elderly population (Regidor et al., 2012). We present results for mortality trends for Spanish male pensioners aged 65 and over since the mid-2000s. We use a large administrative data set (CSWL, the Continuous Sample of Working Lives) to estimate relative differences in mortality among Spanish pensioners grouped according to their initial pension income (PI) levels. We use this initial PI level as our one indicator of socioeconomic status. This approach enables us to accurately answer two basic research questions: (1) are there any differences in mortality between PI income groups? and (2) can any different trends in life expectancy be detected between PI groups that may lead to an increase or a reduction in inequalities over time?

In line with what has been reported for other Mediterranean countries, our paper find that mortality inequalities among older Spanish adults are small. The ubiquity of social safety nets, widespread adherence to the "Mediterranean diet", a later economic modernization process and the existence of better health assets may be responsible for this finding.

We find an inverse relationship between PI levels and mortality for male retirement pensioners. The trend over the full period analysed shows that the spread of life expectancy by PI level has widened. These inequalities are relatively small, but they are also statistically significant. It does not appear that the pension system could be responsible for this slight growth in inequality, given that the Spanish system has been moving away from its 
insurance-based roots towards an increasingly redistributive model. Further research needs to be done on this aspect, but the causes might be found in the decrease in spending on public health and the increased spending on private health during the period 2009-2018.

The rest of the paper is structured as follows: Section 2 describes the population studied and the methodology used to analyze mortality among Spanish pensioners. Section 3 presents the main results. Section 4 discusses some issues arising from the results. The paper ends with concluding comments and a brief technical appendix describing the methodology used to calculate crude death rates, relative mortality ratios, standard errors of life expectancy, and the significance test for checking the robustness of results.

\section{2.-Methods}

\section{1.-Variables and population studied}

As mentioned above, we use a large administrative data set. This has various advantages over the use of survey data, including larger sample sizes, lower costs and a lighter respondent burden. The CSWL is a random sample of around 1.2 million people, i.e. $4 \%$ of the reference population. It contains administrative data on the working lives that form the basis of the sample taken from Spanish Social Security records and comprises anonymized microdata with detailed information on individuals (Pérez-Salamero et al., 2017).

The first wave covers people who had a financial link with the Social Security system in 2004 and provides the entire working history of the sample population. The sample is updated every year using information from variables selected from the Social Security system - dating back to when computerized records began - and from other administrative data sources that record additional information on individuals. The data available to researchers cover from 2004 to 2018. We use the CSWL without fiscal data because it contains a greater number of records than with fiscal data.

The sample reference population is defined as individuals who have had some connection (through contributions, pensions or unemployment benefits) to the Social Security system at any time during the year of reference. Individuals who for any reason have no connection to Social Security in a particular year do not appear in the CSWL. Nor are public employees included.

In our study the initial population comprises male retirement pensioners who retired from the general scheme at age 65 (the ordinary retirement age) or over. Figure 1 provides a flow chart of the participants/records excluded from our study.

Because of lower labour force participation rates among the equivalent female cohorts and the fact that women sometimes have shorter careers (in terms of years of employment) and may work less intensively than men due to family roles and commitments, the PI level is not a suitable indicator for women's working-life income. The focus of our analysis in this research is therefore the social gradient in male mortality (Figure 1, box 3). 


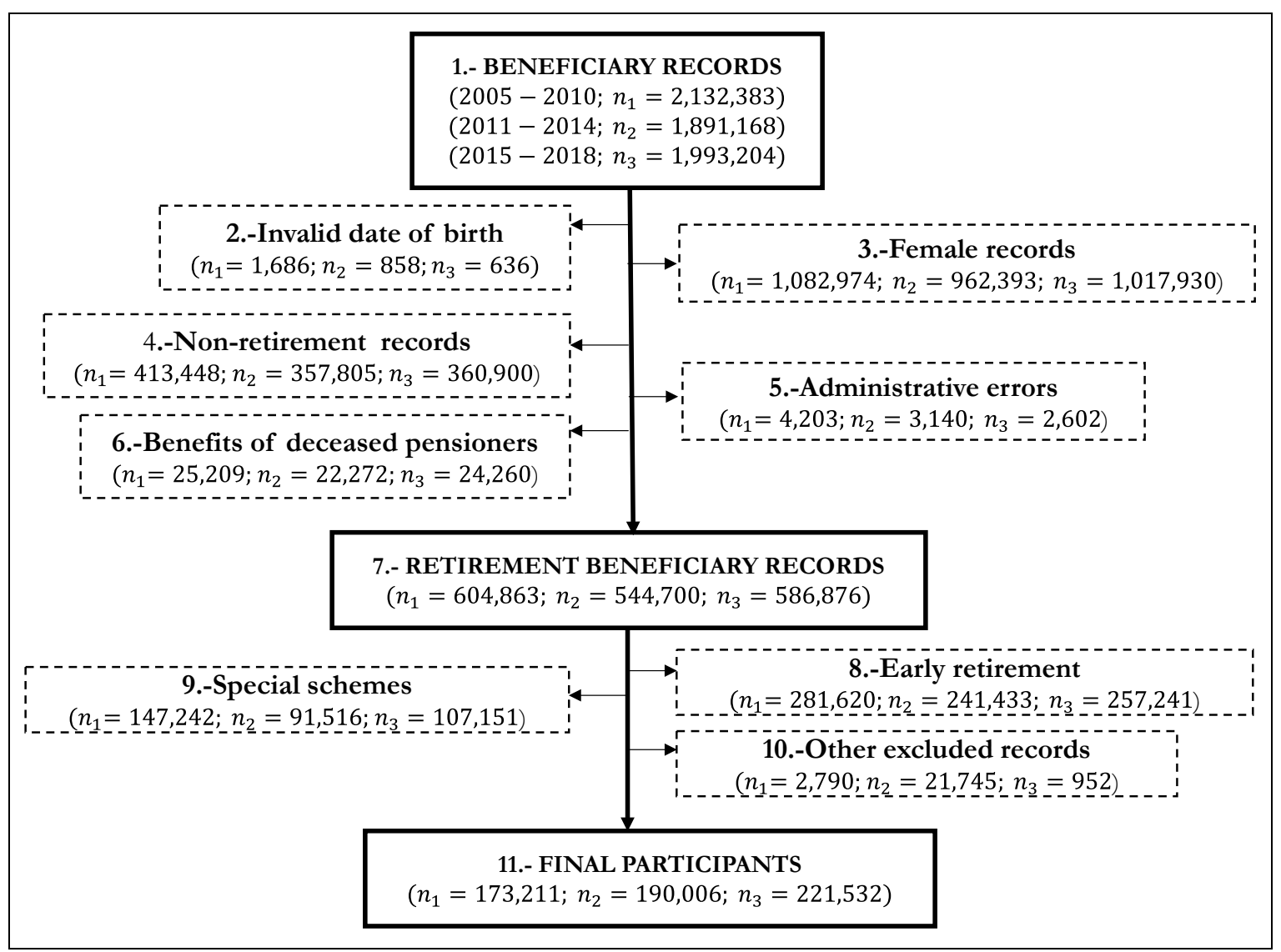

Figure 1: Flow chart showing participants/records excluded from the present study, CSWL, 2005-2018

Mortality among disabled people is far higher than among the general population (Gjesdal et al., 2009; Duran, 2016; Park et al., 2017), so combining the two populations (retirement pensioners and disabled pensioners) could have a seriously misleading effect when it comes to accurately determining the social gradient in mortality (Figure 1, box 4).

Given that poor health is an important reason for early retirement (Wu et al., 2016), pensioners who access benefits before the statutory retirement age are also excluded (Figure 1, box 8).

Retirement pensioners belonging to the special system for the self-employed are also excluded because the pension rules make their benefits a poor proxy for lifetime income (Figure 1, box 9).

Of all the initial beneficiary records for each of the periods considered (box 1), by the end (box 11) - once the whole process of sifting and excluding had been completed - only $8.13 \%$ of the original records remain for the first period, along with $10.05 \%$ for the second and $11.11 \%$ for the third.

The following variables are available in the CSWL data: month and year in which the pension was first paid and ended (if ended), regulating base used to calculate the amount of the benefit, years contributed under each pension regime, benefit type (old age pension, early retirement, disability insurance, survivor's benefits, other) and sex. The design of the Spanish pension system guarantees that retirement benefits are closely linked to lifetime earnings. The contributory system is structured into different "regimes" or schemes, each of which covers a group of workers of a particular type. The General Regime is the basic core of the entire system and includes all employed people over 16 who are not included in any other "special regime." 


\begin{tabular}{|c|c|c|c|c|c|c|}
\hline \multicolumn{7}{|c|}{$\begin{array}{c}\text { Table 1: Hypothetical pensioner income levels: exposures in person-years and } \\
\text { number of deaths by period (absolute values and percentages) }\end{array}$} \\
\hline \multirow[b]{2}{*}{ Periods } & \multirow[b]{2}{*}{ Items } & \multicolumn{4}{|c|}{ Groups } & \multirow{2}{*}{ Total } \\
\hline & & Low & Med-Low & Med-High & High & \\
\hline \multirow{4}{*}{ 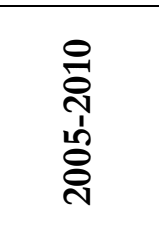 } & Exposures & 22,146 & 70,622 & 26,169 & 28,165 & 147,102 \\
\hline & \% Exposures & 15.05 & 48.01 & 17.79 & 19.15 & 100 \\
\hline & Deaths & 1,132 & 3,379 & 937 & 781 & 6,229 \\
\hline & $\%$ Deaths & 18.17 & 54.25 & 15.04 & 12.54 & 100 \\
\hline \multirow{4}{*}{ 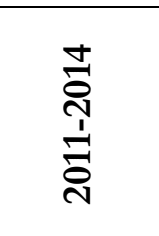 } & Exposures & 20,116 & 74,562 & 23,958 & 37,129 & 155,764 \\
\hline & $\%$ Exposures & 12.91 & 47.87 & 15.38 & 23.84 & 100 \\
\hline & Deaths & 970 & 3,539 & 902 & 874 & 6,285 \\
\hline & $\%$ Deaths & 15.43 & 56.31 & 14.35 & 13.91 & 100 \\
\hline \multirow{4}{*}{ 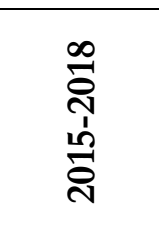 } & Exposures & 21,562 & 83,420 & 25,995 & 49,921 & 180,897 \\
\hline & \% Exposures & 11.92 & 46.11 & 14.37 & 27.60 & 100 \\
\hline & Deaths & 1,009 & 4,003 & 1,040 & 1,138 & 7,190 \\
\hline & $\%$ Deaths & 14.03 & 55.67 & 14.46 & 15.83 & 100 \\
\hline \multicolumn{7}{|c|}{ Dwn work based on CSWL 2005-2018 } \\
\hline
\end{tabular}

Table 1 shows the exposures in person-years and number of deaths (absolute values and percentages) for "hypothetical pensioner income levels" and periods studied. To analyse the data we group the records into four PI levels $\left(B_{m}\right)$ : "1-Low"; "2-Medium-Low", "3Medium-High" and "4-High". We ruled out using benefit level quartiles because, given the features of the sample, some individuals could change quartile due to the appearance of new entrants in each wave.

We assign pensioners to each group according to the minimum (Min) and maximum (Max) benefits in force at the time of their retirement: Low: $B_{1} \leq$ Min; Medium-Low: Min $<$ $B_{2} \leq(0.5 \mathrm{Max}+0.33 \mathrm{Min}) ;$ Medium-High: $(0.5 \mathrm{Max}+0.33 \mathrm{Min})<B_{3} \leq 0.75 \mathrm{Max}$; and High: $B_{4}>0.75 \mathrm{Max}$. Based on the benefit rates in force, the ranges vary over the years reported, e.g. between $€ 36,121.82$ (Max) and $€ 8,727.60$ (Min) per year in 2018. For the 2018 calendar year, the cut-off points between the four PI level intervals are $€ 8,727.60, € 20,679.19$, and $€ 27,091.37$ respectively.

\section{2.-Methodology}

We study the social gradient in mortality in two ways. First we estimate an indicator known as "relative mortality", which we use to compare the graduated death rates among retired male pensioners by age group and PI level with the annual death rate for retired male pensioners in the same age group for three different periods: $P_{1}: 2005-2010, P_{2}: 2011$ 2014 , and $P_{3}: 2015-2018$. The first period covers five years, while the second and third cover four. The actual number of deaths and the risk exposure used to calculate the crude annual death rate come from the CSWL (Table 1).

For each age group we calculate relative mortality rates at various PI levels. A relative mortality rate of 1.00 for any PI level indicates that it is the same as the death rate for that age group as a whole. If it is lower (higher) than 1.00, the death rate for that PI level is lower (higher) than the rate for that age group as a whole (or any other group of interest). (see technical appendix for details)

Second, we estimate the changes in total life expectancy by PI level at age $65\left(\mathrm{LE}_{65}\right)$ over time. To do this we use the Mort1Dsmooth function in the MortalitySmooth $\mathrm{R}$ package 
(Camarda, 2012) - specially designed for use in mortality research - to construct complete period life tables from age 65 to age 101 and to calculate $\mathrm{LE}_{65}$ for each of the three periods analysed. (see technical appendix for details).

\section{3.-Results}

Table 2 shows relative mortality rates by age group and PI level for the three different periods considered, which together cover from 2005 to 2018: $\mathrm{P}_{1}: 2005-2010, \mathrm{P}_{2}$ : 2011-2014 and $\mathrm{P}_{3}$ : 2015-2018.

For 2005-2010 the relative rates for the 65-69 age group are 1.49, 1.03, 0.95 and 0.77 in ascending order from the lowest PI level to the highest. The figure of 1.49 for the lowest means that the death rate is $49 \%$ higher than for that age group as a whole, while the figure of 0.77 for the highest PI level means that it is $23 \%$ lower than for that age group as a whole. Table 2 shows that for groups covering higher ages there is less of a difference in relative mortality rates between PI levels.

If we compare interval $\mathrm{P}_{1}: 2005-2010$ and $\mathrm{P}_{2}: 2011-2014$, we see a generalized increase in relative inequality in mortality for almost all age groups. The exception is the 75-79 age group, where the difference in relative mortality between the lowest and highest PI levels drops from 0.23 (1.12-0.89) to 0.17 (1.11-0.94), i.e. the relative inequality in mortality for this age group is lower than in the previous period.

When we compare interval $\mathrm{P}_{2}$ : 2011-2014 and $\mathrm{P}_{3}$ : 2015-2018, we see a substantial increase in relative inequality in mortality for the youngest age groups (65-69; 70-74 and 75-79) and a slight reduction for the rest (80-84; and $85+)$.

\begin{tabular}{|c|c|c|c|c|c|}
\hline \multirow[b]{2}{*}{ Periods } & \multirow{2}{*}{$\begin{array}{c}\text { Age } \\
\text { Group }\end{array}$} & \multicolumn{4}{|c|}{ PI level } \\
\hline & & Low & Med-Low & Med-High & High \\
\hline \multirow{6}{*}{$\begin{array}{l}\text { 융 } \\
\text { ㄱ. } \\
\text { ம் } \\
\text { రิ }\end{array}$} & $65-69$ & 1.49 & 1.03 & 0.95 & 0.77 \\
\hline & $70-74$ & 1.18 & 1.05 & 0.86 & 0.83 \\
\hline & $75-79$ & 1.12 & 1.04 & 0.92 & 0.89 \\
\hline & $80-84$ & 1.05 & 1.02 & 0.95 & 0.92 \\
\hline & $85+$ & 1.00 & 1.01 & 0.90 & 0.90 \\
\hline & Total & 1.18 & 1.13 & 0.86 & 0.61 \\
\hline \multirow{6}{*}{ 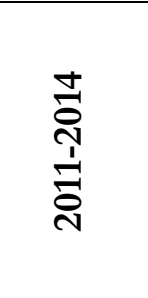 } & 65-69 & 1.59 & 1.02 & 0.94 & 0.85 \\
\hline & $70-74$ & 1.21 & 1.05 & 0.89 & 0.82 \\
\hline & $75-79$ & 1.11 & 1.00 & 0.96 & 0.94 \\
\hline & $80-84$ & 1.06 & 1.03 & 0.95 & 0.84 \\
\hline & $85+$ & 1.05 & 1.05 & 0.89 & 0.81 \\
\hline & Total & 1.21 & 1.15 & 0.80 & 0.59 \\
\hline \multirow{6}{*}{ 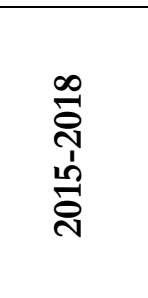 } & $65-69$ & 1.67 & 1.09 & 0.93 & 0.75 \\
\hline & $70-74$ & 1.37 & 1.07 & 0.91 & 0.79 \\
\hline & $75-79$ & 1.22 & 1.07 & 0.95 & 0.85 \\
\hline & $80-84$ & 1.07 & 1.06 & 0.91 & 0.86 \\
\hline & $85+$ & 1.05 & 1.08 & 0.85 & 0.83 \\
\hline & Total & 1.25 & 1.19 & 0.78 & 0.58 \\
\hline
\end{tabular}

Table 2 shows the trend in relative mortality rates for the whole range of age groups and periods analysed. It reveals that the spread of death rates across PI levels has been widening for male retirement pensioners aged 65 and over. 
The decrease in death rates by PI level between intervals $\mathrm{P}_{1}$ and $\mathrm{P}_{3}$ fully explains the change in the relative inequality in mortality. This decrease is (much) larger for the "High" $(14.18 \%)$ and "Medium-High" groups (10.91\%) than for the group as a whole $(8.76 \%)$. Given that the previous relative mortality rates were less than 1.00 for both groups $(0.61$ and 0.86 respectively in $\left.\mathrm{P}_{1}\right)$, the figures for these two groups in the third period $(0.78$ and 0.58 respectively) indicate that inequality in mortality has increased over time. In the case of the "Low" and "Medium-Low" groups the improvements in mortality are (much) smaller $(2.97 \%$ and $3.73 \%$ respectively) than for the group as a whole $(8.76 \%)$, and therefore their rates in this third period (1.25 and 1.19 respectively) are further from 1.00 than they were in the first period (1.18 and 1.13 respectively).

At first glance it might appear that the relative mortality rates observed for the periods analysed would imply enormous differences in life expectancy between the groups of pensioners categorized by PI level. However, as we can see below, this is not strictly true.

Figure 2 shows full life expectancy at age 65 along with several variations by PI level for the periods studied. The information has been broken down into 4 graphs.

Graph 1 in Figure 2 shows that $\mathrm{LE}_{65}$ is positively linked to PI levels. The higher the PI level, the higher the life expectancy at age 65. In Graph 2 we see that for 2005-2010 there is a gap of 1.49 years between pensioners in the lowest and those in the highest income groups. This gap widens over time to 2.58 years in 2015-2018. A similar trend can be observed if the highest PI group is compared to the other two groups. The trend sketched out by the columns points to constantly increasing differences in socioeconomic mortality for all groups, but with a steeper social gradient in the lowest PI group.

Given these results, we now need to find out whether or not the $\mathrm{LE}_{65}$ differences between PI levels are statistically significant. Table 3 and Graph 3 in Figure 2 can shed some light on this matter. 


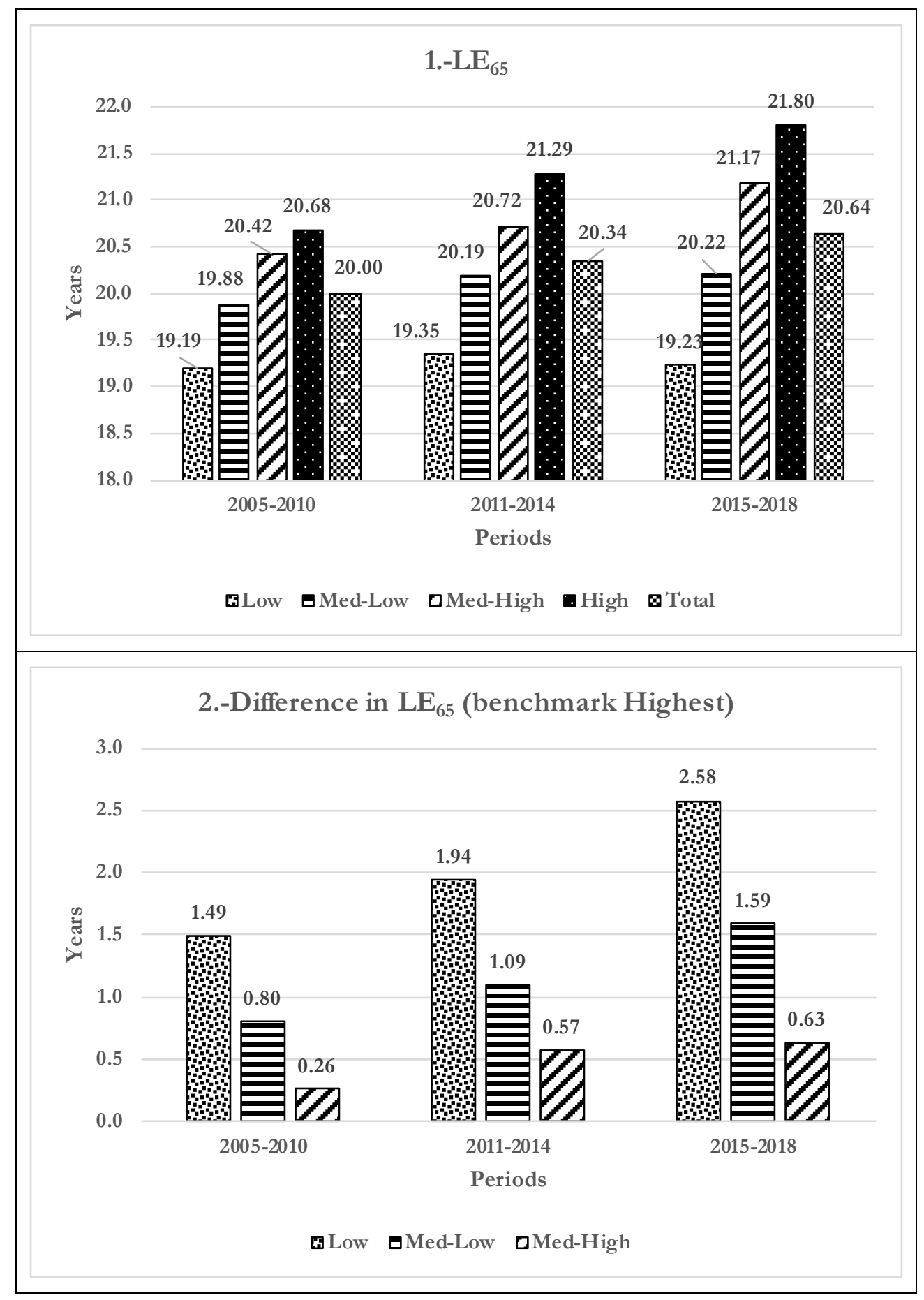




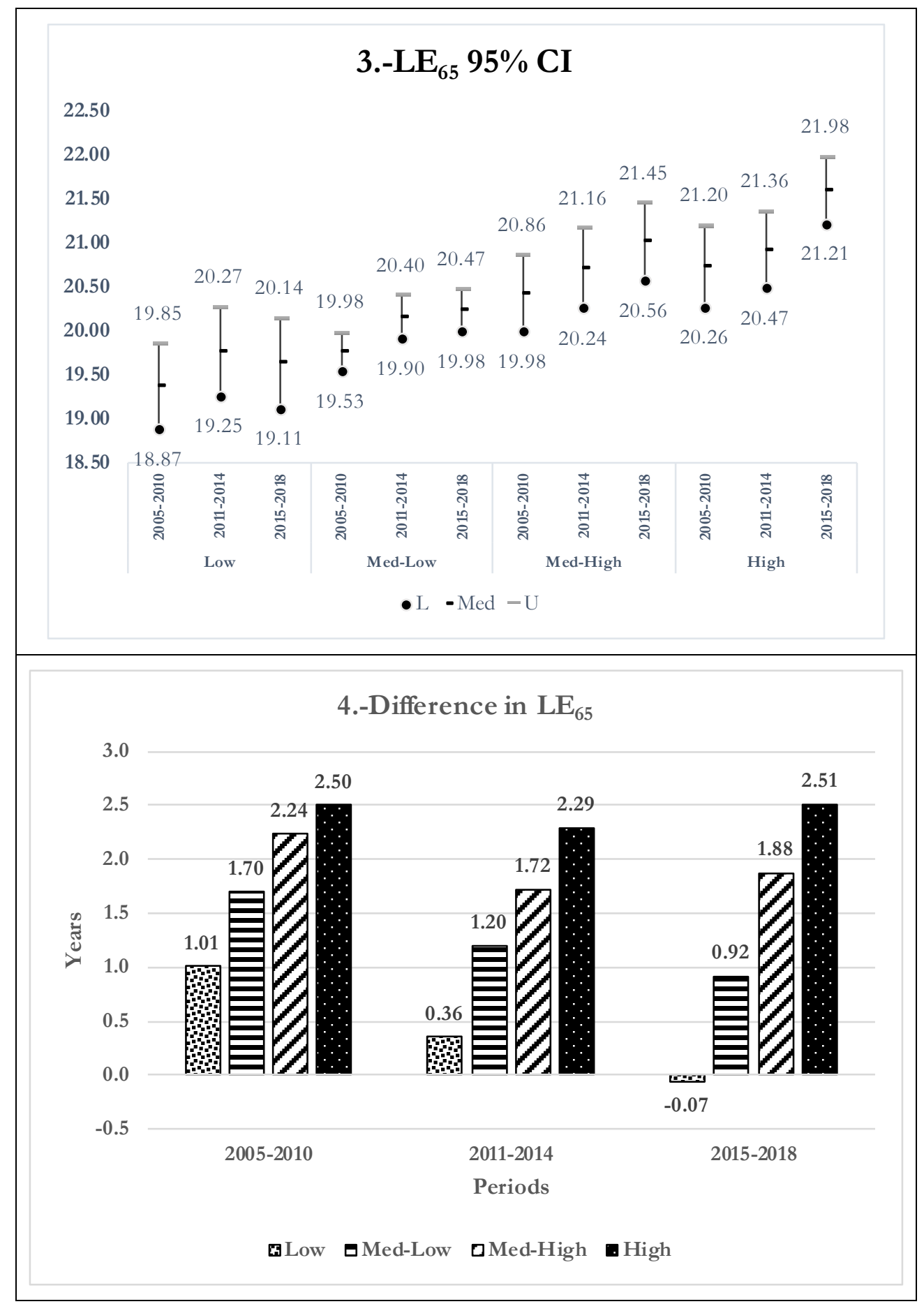

Figure 2: $\mathrm{LE}_{65}$ and variations by PI level. 
For all three periods analysed, Table 3 shows the differences in $\mathrm{LE}_{65}\left(\mathrm{DLE}_{65}\right)$ between one PI group and another, from Med-Low and Low to High and Med-High, along with the standard error for those differences and the z-score of the test statistic. Thus we can test the null hypothesis that the difference in life expectancy is zero against the alternative of its being positive. The results show that most $\mathrm{DLE}_{65}$ are statistically significant at $1 \%$ or $5 \%$, with the sole exception being the difference between the High and Med-High PI groups in 2005-2010, which is not significant at 10\%. These results therefore support the idea that there is highly significant evidence of a positive relationship between $\mathrm{LE}_{65}$ and PI groups.

\begin{tabular}{|c|c|c|c|c|c|c|c|c|c|}
\hline Items & $\begin{array}{r}\text { D } \\
\text { Med-I }\end{array}$ & $\begin{array}{l}\text { f. betw } \\
\text { ow and } \\
\text { groups }\end{array}$ & $\begin{array}{l}\text { en } \\
\text { Low PI }\end{array}$ & $\begin{array}{r}\text { I } \\
\text { Med } \\
\text { L }\end{array}$ & $\begin{array}{l}\text { f. Betw } \\
\text { High ar } \\
\text { v PI gr }\end{array}$ & $\begin{array}{l}\text { en } \\
\text { d Med- } \\
\text { ups }\end{array}$ & $\begin{array}{r}\text { D } \\
\text { High }\end{array}$ & $\begin{array}{l}\text { f. Betw } \\
\text { ind Me } \\
\text { I grou }\end{array}$ & $\begin{array}{l}\text { en } \\
\text {-High } \\
\text { s }\end{array}$ \\
\hline Periods & $\mathbf{P}_{1}$ & $\mathbf{P}_{2}$ & $\mathbf{P}_{3}$ & $\mathbf{P}_{1}$ & $\mathbf{P}_{2}$ & $\mathbf{P}_{3}$ & $\mathbf{P}_{1}$ & $\mathbf{P}_{2}$ & $\mathbf{P}_{3}$ \\
\hline$\overline{D L E}_{65}$ & 0.689 & 0.843 & 1.228 & 0.541 & 0.524 & 0.714 & 0.261 & 0.569 & 0.631 \\
\hline $\operatorname{Se}\left(\mathrm{DLE}_{65}\right)$ & 0.269 & 0.283 & 0.282 & 0.255 & 0.264 & 0.263 & 0.330 & 0.337 & 0.312 \\
\hline z-score & $2.56^{* * *}$ & $2.98^{* * *}$ & $4.36^{* * *}$ & $2.12^{* *}$ & $1.96^{* *}$ & $2.71^{* * *}$ & 0.79 & $1.69^{* *}$ & $2.02^{* *}$ \\
\hline \multicolumn{10}{|c|}{$\begin{array}{l}* * * \text { significant at } 1 \% \text { one-tailed test. } * * \text { significant at } 5 \% \text { one-tailed test. } * \text { significant at } 10 \% \\
\text { one-tailed test. }\end{array}$} \\
\hline \multicolumn{10}{|c|}{ Source: Own work based on CSWL 2005-2018 } \\
\hline
\end{tabular}

The details given in Table 3 can be seen more intuitively in Graph 3, which shows 95\% confidence intervals (CIs) for the $\mathrm{LE}_{65}$ for all PI groups of pensioners and all three periods analysed.

Graph 4 in Figure 2 compares our results with the $\mathrm{LE}_{65}$ for the Spanish population as a whole. As expected, those individuals included in the sample live longer than the general population because one of the requirements for obtaining a retirement pension is to have contributed for at least 15 (currently 20) years, including at least 2 of the last 15 (20) years. This is likely to exclude some of the most at-risk members of the population because of the strong correlation between labour force participation and health observed in various countries (Waldron, 2007; Urbanaviciute et al., 2019; Piłat, 2020). Disability pensioners and early retirees are also excluded. The most striking thing is that $\mathrm{LE}_{65}$ for pensioners in the lowest PI group is actually lower than for the general population in the third period. Absolute differences in life expectancy for the most disadvantaged groups are seen to narrow over time, while for the most advantaged groups they remain fairly stable

\section{4.-Discussion}

We find an inverse relationship between PI levels and mortality for male retirement pensioners. The trend over the full period analysed shows that the spread of life expectancy by PI level has widened. This result is in line with studies on Germany, which have also reported increasing inequalities among elderly men based on pension fund data (Kibele et al., 2013; Tetzlaff et al., 2020; Wenau et al., 2016).

Overall, the literature reviewed (Mackenbach et al., 2016; Longevity Science Panel, 2018, 2020; Waldron et al., 2007; Bosley et al., 2018; Belloni et al., 2013; Lallo and Raitano, 2018; Adam, 2012; Wen et al., 2020; Kibele et al., 2013; Tetzlaff et al., 2020; Wenau et al., 2019; Kalwij et al., 2013) generally indicates that when mortality gaps have widened over time in the past, the probabilities of death have usually decreased more rapidly for high-income groups than for low-income groups. We also find evidence of this for Spain using the data from the CSWL. 
Our findings show that inequalities in mortality for retirement pensioners are small. This is in line with previous findings for Spain involving older adults (Regidor et al., 2012; Kulhánová et al., 2014; Solé-Auró et al., 2020). The ubiquity of social safety nets (Regidor et al., 2012), widespread adherence to the "Mediterranean diet", a later economic modernization process and the existence of better health assets may be responsible for this finding.

Along with behavioural and structural aspects (Mackenbach, 2017), a combination of other factors such as the design of the pension system (EU, 2018), the universality and good quality of the health system (Fullman, 2018) and high levels of family support (LorenzoCarrascosa, 2015) could explain why inequalities in life expectancy for retired Spanish men are relatively small.

Nevertheless, although the differences are small, they have grown. It does not appear that the pension system could be responsible for this growth in inequality, given that the Spanish system has one of the highest aggregate replacement rates in Europe. Spanish pensioners largely maintained their relative standard of living during the recent economic crisis (2008-2014) (EU, 2018), and the percentage of people in Spain aged 65 and above whose income is less than $50 \%$ of median equivalized disposable household income is lower $(9.4 \%)$ than the figure for the total population $(15.5 \%)$. This rate is also 4.1 percentage points below the average for OECD countries $(13.5 \%)$ in 2016 (OECD, 2019b).

The contributory retirement pension system in Spain has been moving away from its insurance-based roots towards an increasingly redistributive model. The minimum retirement pension for a single pensioner at age 65 increased by $15.24 \%$ in real terms between 2000 and 2018 and the maximum benefit decreased by $5.86 \%$ in real terms. The ratio between the maximum and minimum pensions has greatly decreased over time, from 5.07 (2000) to 4.14 (2018). Around 91\% of people aged 65 and over live in owner-occupied homes, and only 1.8\% were found to be living in overcrowded households (EU, 2018).

The Spanish health system is based on the principle of universality and is one of the most efficient healthcare systems in Europe. Indeed, in the Healthcare Access and Quality Index, Spain is ranked $19^{\text {th }}$ out of 195 countries for healthcare and access. Perhaps the reason for this increase in inequality in life expectancy can be found in the lack of investment in the public health system. Over the period 2008-2013, annual per-capita spending on health (in real terms) decreased by $-1.9 \%$, better than only Greece, Portugal and Iceland of the OECD36 countries (OECD, 2019a). Growth during the period 2013-2018 was positive at $2.3 \%$, very close to the average for the period for all the 36 countries of the OECD studied $(2.4 \%)$.

Similarly, the macroeconomic figures for total spending on health fell from $9.4 \%$ of GDP in 2009 to $9.1 \%$ in 2018 , but the variation in the proportions of public and private spending was very different (OECD, 2019a). This could partly explain the increase in inequality, since the socioeconomic groups with bigger pensions would presumably benefit the most from these private health services. Public spending on health fell from $7.1 \%$ of GDP in 2009 to $6.4 \%$ in 2018, while spending on the private health sector rose from $2.3 \%$ to $2.7 \%$ over the same period.

Establishing the reasons behind this increased inequality in life expectancy calls for more research to be carried out. An analysis of all Spanish Social Security records instead of just a sample could shed some light on the matter.

\section{1.-Strengths and limitations}


As far as the authors are aware, this is the first time the CSWL has been used to study the differences in mortality and life expectancy from the socioeconomic point of view of retirement pensioners aged 65 and above. As far as the sample finally selected is concerned, the initial amount of pension seems to be a reliable socioeconomic indicator, as shown by the results obtained. However, some limitations to the study should be taken into account. First, we focused on male mortality alone. Second, we excluded groups of pensioners for whom the application of our socioeconomic indicator might not have been suitable for several reasons (disabled pensioners, early retirees, beneficiaries in special schemes such as the self-employed). Third, due to the fact that they are not included in the database used, we were unable to work with the collective of pensioners belonging to the Régimen de Clases Pasivas (civil servants). Finally, despite the fact that the results we have obtained are coherent, the analysis could be carried out in much greater depth if we had access to all the records held by the Department of Social Security, along with details of any additional sources of income the pensioners may have.

\section{5.-Conclusions}

While acknowledging that using the initial pension income level as the sole indicator of socioeconomic status for the retired population has its limitations, we have found an inverse relationship between PI levels and mortality for male retirement pensioners. We have also found highly significant evidence of a positive relationship between $\mathrm{LE}_{65}$ and pension income.

The trends for the entire period analysed show that the spread of life expectancy as measured by PI levels has widened.

The increased inequality in life expectancy does not appear to stem from the pension system reforms carried out over the period 2011-2013, given that the Spanish system has become more redistributive and the amount of the minimum pensions has clearly increased in real terms over recent years. The causes might be found in the decrease in spending on public health during the period 2009-2018 and the increased spending on private health, which would presumably be of greater benefit to those pensioners with higher incomes.

Independently of the causes of differential mortality, a direct link between PI level and life expectancy has important implications for pension policy. Mortality gaps by socioeconomic status should be taken into account for a variety of issues involving social security schemes, e.g. to establish the eligibility age for retirement pensions and early access to benefits, to compute the annuity factors used to determine initial retirement benefits, and to value liabilities to retirement pensioners.

\section{6.-References}

Adam, L. 2012. The Canadian pensioners mortality table: Information on mortality for the triennial period ending December 31, 2007, with data as at December 31, 2008 Quebec City, QC, Canada: Universite Laval. https://www.cia-ica.ca/docs/defaultsource/2013/213003e.pdf

Bambra C. 2011. "Health inequalities and welfare state regimes: theoretical insights on a public health 'puzzle' ". Journal of Epidemiology and Community Health 65: 740-745. doi: $10.1136 /$ jech.2011.136333

Belloni, M., R. Alessie, A. Kalwij, and C. Marinacci. 2013. "Lifetime income and old age mortality risk in Italy over two decades". Demographic Research 29: 1261-1298. doi: 10.4054/DemRes.2013.29.45 
Bosley, T., M. Morris, and K. Glenn. 2018. Mortality by Career-Average Earnings Level. Actuarial study, 124. Social Security Administration, Office of the Chief Actuary. https://www.ssa.gov/OACT/NOTES/pdf studies/study124.pdf

Camarda, C. G. 2012. "MortalitySmooth: An R Package for Smoothing Poisson Counts with P-Splines". Journal of Statistical Software 50: 1-24. http://www.jstatsoft.org/v50/i01/. doi: $10.18637 /$ iss.v050.i01

Chiang, C. L. 1984. The Life Table and its Applications, Malabar, FL: Robert E. Krieger Publishers.

Duggan, J., R. Gillingham, R., and J. Greenlees. 2008. "Mortality and Lifetime Income: Evidence from U.S. Social Security Records". IMF Economic Review 55: 566-594 doi: $10.1057 /$ imfsp.2008.21

Duran, X., C. Vanroelend, P. Deboosere, and F. G. Benavides. 2016. "Social security status and mortality in Belgian and Spanish male workers". Gaceta Sanitaria 30(4): 293-295. doi: $10.1016 /$ i.gaceta.2016.03.016

EU. 2018. The 2018 Pension Adequacy Report: current and future income adequacy in old age in the EU. https://op.europa.eu/en/publication-detail/-/publication/62f83ed2-782111e8-ac6a-01aa75ed71a1/language-en/format-PDF/source-search

Fullman, N., j. Yearwood, S. M. Abay, C. Abbafati, F. Abd-Allah, J. Abdela, et al. . 2018. "Measuring performance on the Healthcare Access and Quality Index for 195 countries and territories and selected subnational locations: a systematic analysis from the Global Burden of Disease Study 2016". The Lancet 391: 2236-2271. doi: 10.1016/S01406736(18)30994-2

Gjesdal, S., P. Svedberg, J. Hagberg, and K. Alexanderson. 2009. "Mortality among disability pensioners in Norway and Sweden 1990-96: Comparative prospective cohort study". Scandinavian Journal of Public Health 37(2): 168-175. doi: $10.1177 / 1403494808100937$

Huisman, M., A. E. Kunst, O. Andersen, M. Bopp, J. K. Borgan, C. Borrell, G. Costa, P. Deboosere, G. Desplanques, A. Donkin, S. Gadeyne, C. Minder, E. Regidor, T. Spadea, T. Valkonen, and J. P. Mackenbach. 2004. "Socioeconomic inequalities in mortality among elderly people in 11 European populations". Journal of Epidemiology and Community Health 58: 468-475. doi: $10.1136 /$ jech.2003.010496

Kalwij, A., R. Alessie, and M. Knoef. 2013. "The association between individual income and remaining life expectancy at the age of 65 in the Netherlands". Demography 50(1): 181206. doi: $10.1007 / \mathrm{s} 13524-012-0139-3$.

Kibele, E. U. B., D. Jasilionis, and V. M. Shkolnikov. 2013. "Widening socioeconomic differences in mortality among men aged 65 years and older in Germany". Journal of Epidemiology and Community Health 67(5): 453-457. doi: 10.1136/jech-2012-201761

Kulhánová, I., A. Bacigalupe, T. A. Eikemo, C. Borrell, E. Regidor, S. Esnaola, J. P. Mackenbach, and Eurothine consortium. 2014. "Why does Spain have smaller inequalities in mortality? An exploration of potential explanations". European journal of public bealth 24(3): 370-377. doi: $10.1093 /$ eurpub/cku006

Lallo, C., and M. Raitano. 2018. "Life expectancy inequalities in the elderly by socioeconomic status: evidence from Italy". Population bealth metrics 16(1): 7. doi: $10.1186 / \mathrm{s} 12963-018-0163-7$

$\mathrm{Li}$, N. 2015. "The probabilistic life table and its applications to Canada". Canadian Studies in Population 42(1-2): 117-129. doi: 10.25336/P65W28 
Longevity Science Panel. 2018. "Life expectancy: Is the socio-economic gap narrowing?" Accessed April 16, 2020. http://www.longevitypanel.co.uk/viewpoint/life-expectancy-isthe-socio-economic-gap-narrowing/.

Longevity Science Panel. 2020. "An analysis of mortality trends in developed countries, focusing on the recent slowdown in mortality improvements". Accessed April 16, 2020. https://www.longevitypanel.co.uk/ files/LSP2020 Report.pdf

Lorenzo-Carrascosa, L. 2015. "Ageing Population and Family Support in Spain". Journal of Comparative Family Studies 46 (4): 499-516. doi: 10.3138/jcfs.46.4.499

Mackenbach, J. P. 2017. "Persistence of social inequalities in modern welfare states: Explanation of a paradox". Scandinavian Journal of Public Health 45: 113-120.

Mackenbach J. P., I. Stirbu, and A. J. Roskam, M. M. Schaap, G. Menvielle, M. Leinsalu, and A. E. Kunst. 2008. "Socioeconomic inequalities in health in 22 European countries". New England Journal of Medicine 358: 2468-2481.

Mackenbach, J. P., M. Bopp, P. Deboosere, K. Kovacs, M. Leinsalue, P. Martikainen, G. Menvielle, E. Regidor, and R. de Gelder. 2017. "Determinants of the magnitude of socioeconomic inequalities in mortality: A study of 17 European countries". Health \& Place 2017; 47: 44-53. doi: 10.1016/i.healthplace.2017.07.005

Mackenbach, J.P., I. Kulhánová, B. Artnik, M. Bopp, C. Borrell, T. Clemens, G. Costa, C. Dibben, R. Kalediene, O. Lundberg, P. Martikainen, G. Menvielle, O. Östergren, R. Prochorskas, M. Rodríguez-Sanz, B. H. Strand, C. W. N. Looman, and R. de Gelder. 2016. "Changes in Mortality Inequalities over Two Decades: Register Based Study of European Countries". British Medical Journal 352(5): 1732. doi: 10.1136/bmj.i1732

OECD. 2019a. Health at a Glance 2019: OECD Indicators, OECD Publishing, Paris. doi:10.1787/4dd50c09-en

OECD. 2019b. Pensions at a Glance 2019: OECD and G20 Indicators, OECD Publishing, Paris, doi: $10.1787 / \mathrm{b} 6 \mathrm{~d} 3 \mathrm{dcfc}-\mathrm{en}$.

Park, J.-M., O. Ukchan, R. Beop-Rae, and M. Yeongmin. 2017. "Disparities in mortality by disability: an 11-year follow-up study of 1 million individuals". International Journal of Public Health 62: 969-996. doi: $10.1007 / \mathrm{s} 00038-017-0966-5$

Pérez-Salamero Gonzalez, J. M., M. Regúlez-Castillo, and C. Vidal-Meliá. 2017. "The continuous sample of working lives: improving its representativeness". SERIEs. Journal of the Spanish Economic Association 8: 43-95. doi: 10.1007/s13209-017-0154-0

Piłat, A., A. Galaś, M. Wilga, M. Cabello, S. Koskinen, J. M. Haro, M. Leonardi, and B. Tobiasz-Adamczyk. 2020. "Gender perspective in the analysis of the relationship between health and work cessation, and how to deal with it". International Journal of Occupational Medicine and Environmental Health 33(3): 365-384. doi: 10.13075/ijomeh.1896.01351

Regidor, E. A. E. Kunst, F. Rodríguez-Artalejo, and J.P. Mackenbach (2012). "Small socioeconomic differences in mortality in Spanish older people". European Journal of Public Health 22: 80-85. doi: 10.1093/eurpub/ckr051

Scherbov, S., and D. Ediev. 2011. "Significance of life table estimates for small populations: Simulation based study of standard errors". Demographic Research 24: 527-550. doi: 10.4054/DemRes.2011.24.22

Solé-Auró, A.; U. Martín, and A. Domínguez Rodríguez. 2020. "Educational Inequalities in Life and Healthy Life Expectancies among the 50-Plus in Spain". International Journal of Environmental Research and Public Health 17: 3558. doi: 10.3390/ijerph17103558 
Tetzlaff, F., J. Epping, S. Sperlich, and J. Tetzlaff. 2020. "Widening income inequalities in life expectancy? Analysing time trends based on German health insurance data". Journal of Epidemiology and Community Health Apr 10. pii: jech-2019-212966. doi: 10.1136/jech-2019212966

Urbanaviciute, I., H. De Witte, and J. Rossier. 2019. "Perceived job insecurity and selfrated health: testing reciprocal relationships in a five-wave study". Social Science Medicine 233: 201-207. doi: 10.1016/j.socscimed.2019.05.039

Waldron, H. 2007. "Trends in Mortality Differentials and Life Expectancy for Male Social Security-Covered Workers, by Socioeconomic Status". Social Security Bulletin 67(3): 128. https://www.ssa.gov/policy/docs/ssb/v67n3/v67n3p1.html

Wen, J., T. Kleinow, and A. J. G. Cairns. 2020. "Trends in Canadian Mortality by Pension Level: Evidence from the CPP and QPP”. North American Actuarial Journal. doi: $\underline{10.1080 / 10920277.2019 .1679190}$

Wenau, G., P. Grigoriev, and V. Shkolnikov. (2019). "Socioeconomic Disparities in Life Expectancy Gains among Retired German Men, 1997-2016". Journal of Epidemiology and Community Health 73: 605-611. doi: 10.1136/jech-2018-211742

Wu, C., M. C. Odden, M. C., G. Fisher, and R. S. Stawski. 2016. "Association of retirement age with mortality: a population-based longitudinal study among older adults in the USA". Journal of Epidemiology and Community Health 70(9): 917-923. doi: 10.1136/jech-2015-207097 


\section{Appendix: Technical Appendix}

\section{A1.-Mortality rates}

Mortality rates (or probabilities of dying) are indicators commonly used in demographics, medicine, and actuarial practice. They usually refer to one or more variables, the most common being age, calendar years, and duration. To describe the actual but unknown mortality pattern of a population (retirement pensioners grouped by initial PI levels and more variables in our case), it is necessary to calculate the crude mortality rates from raw data.

For all beneficiary groups classified by PI level $m$, the crude mortality rate for a given period-year interval, $\mathrm{P}=\{a, a+1, \cdots, n\}$, age $x$, and sex $j$, is defined as the observed probability that a person of age $x$ nearest birthday will die between ages $x$ and $x+1$ during the period-year interval P. $n$ represents $31^{\text {st }}$ December for the last calendar year within the period and $a$ represents $31^{\text {st }}$ December for the first year.

The observed probabilities of death is calculated by simply dividing the relevant number of deaths ${ }^{1}\left(D_{x, \mathrm{P}}^{j, m}\right)$ by the number of life-years of exposure over the given year or period $\left(E_{x, \mathrm{P}}^{j, m}\right)$. The size of the exposure population is estimated by averaging the population sizes at the beginning and end of the year. In our case, the crude mortality rate $\hat{q}_{x, \mathrm{P}}^{j, m}$ is calculated as follows:

$$
\hat{q}_{x, \mathrm{P}}^{j, m}=\frac{D_{x, \mathrm{P}}^{j, m}}{E_{x, \mathrm{P}}^{j, m}}=\frac{\overbrace{D_{x, a+1}^{j, m}+\cdots+D_{x, n}^{j, m}}^{\text {Deaths }}}{\underbrace{\frac{1}{2}\left(D_{x, a+1}^{j, m}+. .+D_{x, n}^{j, m}+L_{x, a}^{j, m}+L_{x, n}^{j, m}\right)+L_{x, a+1}^{j, m} . .+L_{x, n-1}^{j, m}}_{\text {Exposed to Risk }}}
$$

where, $D_{x, \mathrm{t}}^{j, m}$ is the observed number of deaths of individuals who have attained age $x$ on their nearest birthday for PI level group $m$, gender $j$ in the calendar year $t \in\{a+$ $1, \cdots, n\}$, and $L_{x, \mathrm{t}}^{j, m}$, with $t \in\{a, \ldots . n\}$ is the observed number of retirement pensioners aged $x$ at their nearest birthday in PI level group $m$ and gender $j$, at the end of year $t \in \mathrm{P}$.

The average crude death rate $\hat{q}_{h, \mathrm{P}}^{j, m}$ for age group $h$, PI level $m$, gender $j$ for the periodyear interval $\mathrm{P}$ can be expressed as:

$$
\hat{q}_{h, \mathrm{P}}^{j, m}=\frac{\overbrace{D_{h, a+1}^{j, m}+\cdots+D_{h, n}^{j, m}}^{D_{h, \mathrm{P}}^{j, m}}}{\underbrace{\frac{1}{2}\left(D_{h, a+1}^{j, m}+\cdots+D_{h, n}^{j, m}+L_{x, m}^{j, m}+L_{x, n}^{j, m}\right)+L_{x, a+1}^{j, m}+\ldots+L_{x, n-1}^{j, m}}_{E_{h, \mathrm{P}}^{j, m}}}
$$

where $D_{h, t}^{j, m}$ is the observed number of deaths for age group $P$, PI level $m$, gender $j$ in calendar year $t$, and $L_{h, t}^{j, m}$ is the registered number of retirement pensioners in age group $h$, PI level $m$, gender $j$, at the end of year $t \in \mathrm{P}$.

\footnotetext{
${ }^{1}$ Death rates are estimated using observed numbers of deaths and exposures of population. Death counts are inherently random, leading to sampling variation in estimated death rates.
} 
It is straightforward to see that the average crude death rate can also be calculated as a weighted average of the crude death rates for the beneficiaries with ages included in age group $h$, with the weighting being the number of pensioners-years exposed to risk of death:

$$
\hat{q}_{h, \mathrm{P}}^{j, m}=\frac{\sum_{\forall x \in h} \hat{q}_{x, \mathrm{P}}^{j, m} \cdot E_{x, \mathrm{P}}^{j, m}}{\sum_{\forall x \in h} E_{x, \mathrm{P}}^{j, m}}=\frac{\sum_{\forall x \in h} \hat{q}_{x, \mathrm{P}}^{j, m} \cdot E_{x, \mathrm{P}}^{j, m}}{E_{h, \mathrm{P}}^{j, m}}
$$

where $E_{h, \mathrm{P}}^{j, m}$ is the number of pensioners exposed to risk included in age group $h$, PI level $m$, gender $j$, for the period-year interval $\mathrm{P}$.

Similarly, if all PI levels are considered the average crude death rate $\hat{q}_{h, \mathrm{P}}^{j, T}$ for the pensioners in age group $h$, sex $j$ for the period-year interval $\mathrm{P}$ can be expressed as:

$$
\hat{q}_{h, \mathrm{P}}^{j, T}=\frac{\sum_{m=1}^{s} \hat{q}_{h, \mathrm{P}}^{j, m} \cdot E_{h, \mathrm{P}}^{j, m}}{\sum_{m=1}^{s} E_{h, \mathrm{P}}^{j, m}}=\frac{\sum_{m=1}^{s} \hat{q}_{h, \mathrm{P}}^{j, m} \cdot E_{h, \mathrm{P}}^{j, m}}{E_{h, \mathrm{P}}^{j, T}}
$$

where $E_{h, \mathrm{P}}^{j, T}$ is the number of beneficiaries exposed to risk included in age group $h$, at any PI level $m$, gender $j$, for the period-year interval P.

Given that the levels of exposure are not sufficiently high for some groups (see Subsection 2.1), the initial estimates must be revised to produce smoother estimates (graduated mortality rates) using a procedure called graduation ${ }^{2}$. In our case the average crude death rates are graduated through the age, sex, PI pension, and period dimensions to reflect a compromise between smoothness and fit.

The final graduated beneficiary mortality rates, $q_{x, \mathrm{P}}^{j, m}$, represent the best estimates of the rates for the period-year interval P. Once the graduation process has been concluded, the relative mortality ratio, $R M_{h, \mathrm{P}}^{j, m}$, can be obtained immediately. This is the ratio between the death rate of a subgroup and the death rate of the group as a whole. For the subgroup of pensioners in the age interval $h$, with PI level $m$, gender $j$, for the period-year interval P it is:

$$
R M_{h, \mathrm{P}}^{j, m}=\frac{q_{h, \mathrm{P}}^{j, m}}{q_{h, \mathrm{P}}^{j, T}}
$$

\section{A2.-Life expectancy}

Once graduated death rates are obtained it is easy to construct complete period life tables (LT) from age 65 to age 101 and calculate life expectancy at several ages. Life expectancy, $L E_{x}$, is an estimate of the average number of additional years that a person of a given age, $x$, can expect to live. This measure of remaining life is the ratio between total number of person-years lived by the cohort from age $x$ until all members of the cohort have died $\left(T_{x}\right)$ and the number of persons alive at age $x\left(l_{x}\right)$. For complete $L E_{x}$ half a year is added.

\footnotetext{
${ }^{2}$ Graduation has two basic characteristics: smoothness and goodness of fit to the observed data. These two characteristics are in competition and to achieve one of them it is necessary to sacrifice the other.
} 
According to $\mathrm{Li}$ (2015), one important purpose of measuring mortality is to detect differences between populations. To that end, deterministic life tables could specify whether a life-table variable for one population is bigger than the same variable for another population, while a probabilistic life table can further test whether such differences are statistically significant or may appear merely by random chance.

Following Chiang (1984), an observed $L E_{x}$ is a sample mean of future lifetime. Therefore, statistical tests based on normal distribution can be used to draw inferences in comparing $L E$ between two groups for a given age and period $\left(L E_{x, \mathrm{P}}^{m}\right)$. We are interested here in testing whether there is a significant positive difference in $L E_{x, \mathrm{P}}^{m}$ between two pensioner income groups, one with higher PI than the other.

Under the null hypotheses this difference will be zero and under the alternative it will be positive, so it is a one-tailed test. The statistic to be used, the $Z$ score, is the ratio of the difference in $L E$ between the groups $\left(D L E_{x, \mathrm{P}}^{m_{i}-m_{j}}\right)$ to the standard error of that difference $\operatorname{Se}\left(D L E_{x, \mathrm{P}}^{m_{i}-m_{j}}\right)$, which is computed as the square root of the sum of the variances of the corresponding life expectancy at age $x$ for each group, $V L E_{x, \mathrm{P}}^{m_{i}}$ and $V L E_{x, P}^{m_{j}}$ respectively.

$$
z_{x, \mathrm{P}}^{m_{i}-m_{j}}=\frac{D L E_{x, \mathrm{P}}^{m_{i}-m_{j}}}{S e\left(D L E_{x, \mathrm{P}}^{m_{i}-m_{j}}\right)}=\frac{D L E_{x, \mathrm{P}}^{m_{i}-m_{j}}}{\sqrt{V L E_{x, \mathrm{P}}^{m_{i}}+V L E_{x, \mathrm{P}}^{m_{j}}}}
$$

By analogy with the development by Chiang (1984), the standard error for life expectancy at age $x, \operatorname{Se}\left(L E_{x, \mathrm{P}}^{m}\right)$, can be calculated as follows ${ }^{3}$ :

$$
\operatorname{Se}\left(L E_{x, \mathrm{P}}^{m}\right)=\sqrt{\sum_{k=0}^{w-1-x}\left[\left({ }_{k} p_{x, \mathrm{P}}^{m}\right)^{2} \cdot\left(L E_{x+k+1, \mathrm{P}}^{m}\right)^{2} \cdot \frac{\left(q_{x+k, \mathrm{P}}^{m}\right)^{2} \cdot\left(1-q_{x+k, \mathrm{P}}^{m}\right)}{D_{x+k, \mathrm{P}}^{m}}\right]}
$$

where ${ }_{k} p_{x, \mathrm{P}}^{m}$ is the is the probability of surviving from age $x$ to age $\mathrm{x}+\mathrm{k}, q_{x+k, \mathrm{P}}^{m}$ is the probability that an individual aged $x+k$ will die within the year, $L E_{x+k+1, \mathrm{P}}^{m}$ is life expectancy at age $x+k$, and $D_{x+k, \mathrm{P}}^{m}$ is the number of deaths at age $\mathrm{x}+\mathrm{k}$. These elements refer to period $\mathrm{P}$ and PI group $m$.

At a given level of significance $\alpha$, the null hypothesis is rejected if the sample value of the statistic $z_{x, \mathrm{P}}^{m_{i}-m_{j}}$ is greater than the critical value in the normal distribution corresponding to that level of significance $(2.33$ at $1 \%, 1.64$ at $5 \%$, and 1.28 at $10 \%)$. If that is the case, there is statistically significant evidence that $L E_{x, \mathrm{P}}^{m}$ is greater for the higher PI group than for the other.

Finally, it is worth indicating that $95 \%$ confidence intervals (CIs) of $L E_{x, \mathrm{P}}^{m}$ are determined by:

$$
95 \% C I\left(L E_{x, \mathrm{P}}^{m}\right)=L E_{x, \mathrm{P}}^{m} \pm 1.96 \cdot \operatorname{Se}\left(L E_{x, \mathrm{P}}^{m}\right)
$$

\footnotetext{
3 According to Scherbov and Ediev (2011), Chiang's method may be used without significant problems with population sizes from about 10,000 upwards.
} 